\title{
ORGANIZAÇÃO SUPRAMOLECULAR DA FTALOCIANINA DE COBALTO(II) E SEU EFEITO NA OXIDAÇÃO DO AMINOÁCIDO CISTEÍNA
}

\author{
Amanda Costa Santos, Roberto A. Sousa Luz, Luana G. França Ferreira, José Ribeiro dos Santos Júnior e Welter \\ Cantanhêde da Silva* \\ Departamento de Química, Centro de Ciências da Natureza, Universidade Federal do Piauí, 64049-550 Teresina - PI, Brasil \\ Frank Nelson Crespilho \\ Centro de Ciências Naturais e Humanas, Universidade Federal do ABC, 09210-170 Santo André - SP, Brasil
}

Recebido em 13/2/09; aceito em 9/9/09; publicado na web em 23/2/10

\begin{abstract}
SUPRAMOLECULAR ORGANIZATION OF COBALT (II) PHTHALOCYANINE ON THE PATHWAY OF CYSTEINE OXIDATION. The interest in the chemistry of cobalt (II) tetrasulfonated phthalocyanine ( $\mathrm{PcTs} \mathrm{Co}$ ) comes mainly from its macrocycle-ligand structure combined with their special chemical characteristics, such as high solubility, well-defined redox reactions and remarkable optical absorption in the visible region. In this work, we use layer-by-layer technique in order to assemble CoTsPc and poly(allylaminehydrochloride) (PAH) in hybrid supramolecular system. The electronic spectroscopy and cyclic voltammetry techniques were utilized to study PAH/CoTsPc multilayers growth and the cysteine catalytic oxidation. PAH/CoTsPc showed high electrochemical stability and worthwhile to mention is the remarkable influence of supramolecular arrangement on the final redox properties of the system.
\end{abstract}

Keywords: cobalt phthalocyanine; cysteine; supramolecular.

\section{INTRODUÇÃO}

Diversos tipos de materiais nanoestruturados podem ser estudados mediante técnicas de deposição de filmes ultrafinos, ${ }^{1,2}$ principalmente as nanoarquiteturas híbridas. ${ }^{1-3}$ Pode-se citar a técnica Langmuir-Blodgett (LB), onde camadas moleculares são preparadas em ambientes com elevado controle molecular. ${ }^{1,-6}$ Nos filmes LB, as estruturas são extremamente organizadas e podem interagir fortemente com um substrato sólido (vidro, quartzo, silício, ouro etc). Uma vez controlados os parâmetros de deposição, diversas estruturas podem ser idealizadas através da deposição de multicamadas de vários compostos, ${ }^{1,4}$ como biomoléculas, complexos de coordenação, polímeros sintéticos e condutores, cristais líquidos poliméricos, entre outros. Em alternativa à técnica $\mathrm{LB}, \mathrm{Decher}^{7}$ e seus colaboradores ${ }^{8}$ desenvolveram a técnica de automontagem camada-por-camada (ou Layer-by-Layer, LbL), onde interações eletrostáticas de camadas moleculares com cargas opostas governam o equilíbrio de crescimento dos filmes. ${ }^{7,8} \mathrm{~A}$ automontagem ganhou atenção especial devido a vantagens tais como fácil manipulação, baixo custo operacional, reprodutibilidade, versatilidade dos materiais utilizados e elevado controle da arquitetura molecular., ${ }^{2,9-20}$ Diversos materiais têm sido empregados na técnica LbL visando construção de filmes nanoestruturados, como metaloftalocianinas, ${ }^{1,2,11-31}$ porfirinas, ${ }^{32-34}$ polímeros condutores, ${ }^{1,10}$ diazo-resinas, ${ }^{15}$ nanopartículas metálicas ${ }^{3,16,35}$ e moléculas biológicas. ${ }^{14,16,17} \mathrm{Em}$ especial, compostos de coordenação, quando imobilizados em filmes LbL, apresentam comportamento químico diferenciado quando comparados em solução e no estado sólido, situações nas quais efeitos supramoleculares devem ser levados em consideração..$^{2,12,32,34,36}$ Neste sentido, as propriedades químicas e físicas de diversas arquiteturas supramoleculares ${ }^{32,33,37,38}$ puderam ser melhor interpretadas por meio do entendimento da química supramolecular, que utiliza conhecimentos de processos de auto-organização e de interações específicas. ${ }^{2,32-34,37-39}$

Recentemente, Lehn $^{40}$ reportou que os componentes químicos

*e-mail: welter@ufpi.edu.br que interagem em nível supramolecular podem ser estudados através da Química Dinâmica Constitucional (QDC), uma vez que as interações intermoleculares são vulneráveis e, ademais, há certo grau de reversibilidade e orientação das ligações covalentes dentro da arquitetura molecular. Face à complexidade da estrutura química de arquiteturas híbridas, a QDC abre uma grande possibilidade de interpretação e entendimento das propriedades e reatividades de arranjos mais sofisticados proporcionando, consequentemente, um avanço da nanotecnologia molecular. ${ }^{34}$ Em estudos recentes, ${ }^{41}$ mostramos que o conceito de QDC pode ser aplicado quando ftalocianinas metálicas são incorporadas em filmes LbL. Por exemplo, após a imobilização de nanopartículas de ouro (AuNP) em filmes LbL supramoleculares contendo FtTsNi (PAH-AuNP/ FtTsNi), observou-se um aumento de aproximadamente $80 \%$ das correntes faradaicas para os processos redox da ftalocianina e do metal $\left(\mathrm{Ni}^{2+} / \mathrm{Ni}^{3+}\right) .{ }^{41}$ Além disso, apenas o eletrodo modificado ITO(PAH-AuNP/FtTsNi) ${ }_{3}$ provocou um efeito catalítico na oxidação de peróxido de hidrogênio, com aumento das correntes anódica e catódica para ambos os processos redox, diferentemente do observado pelo eletrodo ITO-(PAH/FtTsNi) ${ }_{3}$ (sem AuNP), onde apenas o primeiro processo redox foi afetado..$^{41}$

Geralmente, ftalocianinas tetrassulfonadas de cobalto II (FtTsCo) $)^{20,25,28,42-44}$ imobilizadas em filmes finos LbL apresentam processos redox bem definidos, elevada estabilidade química e um arranjo geométrico quadrado planar, onde os grupos pirrois, no plano equatorial, apresentam-se inertes em relação às reações de substituição. ${ }^{2,45-47}$ Além disso, as FtTsCo têm sido utilizadas para construção de sensores para moléculas biológicas e catalisadores de diversas espécies, como glutationa (GSH), ${ }^{22,23,48}$ cisteína (CiSH), ${ }^{23-27,48}$ histidina, ${ }^{26}$ monóxido de nitrogênio, ${ }^{21,28,31}$ pesticidas, ${ }^{29}$ glucose ${ }^{30}$ e hidrazina. ${ }^{31}$

Desta forma, este trabalho apresenta as propriedades supramoleculares de filmes multicamadas contendo FtTsCo(II) e polialilamina (PAH) (PAH/FtTsCo). Utilizou-se a voltametria cíclica e a espectroscopia eletrônica na região do visível como técnicas de investigação. Por fim, avaliaram-se as propriedades eletrocatalíticas do sistema $\mathrm{PAH} / \mathrm{FtTsCo}$ para oxidar o aminoácido cisteína. 


\section{PARTE EXPERIMENTAL}

\section{Materiais e reagentes}

Ftalocianina tetrassulfonada de cobalto II (FtTsCo) e cloreto de polialilamina $(\mathrm{PAH})$ tiveram procedência Aldrich. Os ácidos sulfúrico $\left(\mathrm{H}_{2} \mathrm{SO}_{4}\right)$ e clorídrico $(\mathrm{HCl})$, procedência Reagen, foram utilizados na preparação das soluções com concentrações de $3,2 \times 10^{-3}(\mathrm{pH} 2,5)$ e $0,10(\mathrm{pH} 1,0) \mathrm{mol} \mathrm{L}^{-1}$, respectivamente, sendo que o $\mathrm{H}_{2} \mathrm{SO}_{4}$ foi usado como solvente e o $\mathrm{HCl}$ como eletrólito suporte. Di-hidrogeno fosfato de potássio $\left(\mathrm{KH}_{2} \mathrm{PO}_{4}\right)$, procedência Reagen, e hidróxido de sódio $(\mathrm{NaOH})$, procedência Vetec, foram utilizados na preparação da solução de 0,1 mol L-1 $\mathrm{KH}_{2} \mathrm{PO}_{4}$ (PBS) pH 7,0. O aminoácido cisteína, procedência Aldrich, foi utilizado no teste biocatalítico. Todas as soluções foram preparadas com água purificada por meio do sistema Millipore (Milli-Q).

\section{Automontagem das arquiteturas supramoleculares}

Para construção dos filmes LbL, utilizaram-se soluções de FtTsCo $\left(0,5 \mathrm{~g} \mathrm{~L}^{-1}\right)$ e PAH $\left(0,5 \mathrm{~g} \mathrm{~L}^{-1}\right)$ em pH 2,5 ( $\left.\mathrm{H}_{2} \mathrm{SO}_{4}\right)$. Na fabricação do filme LbL, o substrato foi imerso na solução de PAH por 5 min, onde uma camada carregada positivamente foi adsorvida; depois, enxaguado por 10 s em solução de $\mathrm{H}_{2} \mathrm{SO}_{4} \mathrm{pH} 2,5$ e seco com fluxo de $\mathrm{N}_{2}(99,9 \%)$. Posteriormente, o mesmo substrato foi imerso na solução de FtTsCo, onde uma camada carregada negativamente foi adsorvida, enxaguado novamente por $10 \mathrm{~s}$ em outra solução de $\mathrm{H}_{2} \mathrm{SO}_{4} \mathrm{pH} 2,5$ e seco com fluxo de $\mathrm{N}_{2}$, formando o filme PAH/FtTsCo com uma bicamada. A repetição cíclica deste processo promove a formação da arquitetura supramolecular com o número desejável de bicamadas (Figura 1). As diferentes arquiteturas supramoleculares para o sistema (PAH/FtTCo) (onde $n$ representa o número de bicamadas de 1 a 20) foram construídas sobre substratos de vidro (BK7) e vidro coberto com óxido de estanho dopado com índio (ITO) ${ }^{1,11-19}$ utilizando o método LbL. ${ }^{7}$ Os substratos foram previamente limpos por meio do processo de hidrofilização. ${ }^{49}$

\section{Caracterização dos filmes LbL}

O acompanhamento do crescimento dos filmes LbL de PAH/ FtTsCo e o estudo do comportamento da FtTsCo em solução foram realizados através de um espectrofotômetro Hitachi U-3000. Para os estudos voltamétricos, utilizou-se uma cela eletroquímica $(10 \mathrm{~mL})$, com três eletrodos, acoplada a um potenciostato Autolab PGSTAT 30 Eco Chemie. Eletrodos de calomelano saturado (ECS) e platina (área $=1,0 \mathrm{~cm}^{2}$ ) foram utilizados como referência e contra eletrodo, respectivamente. Como eletrodo de trabalho, usou-se ITO não modificado ou ITO modificado por FtTsCo ou PAH/FtTsCo (área = 0,35 $\mathrm{cm}^{2}$ ). Antes de registrar os voltamogramas cíclicos do sistema (PAH/ FtTsCo), removeu-se o oxigênio do eletrólito através da passagem de um fluxo de $\mathrm{N}_{2}(99,9 \%)$ durante $10 \mathrm{~min}$.

O estudo eletroquímico para ITO-(PAH/FtTsCo) foi realizado em função do número de bicamadas depositadas $(n)$, aumento da velocidade de varredura, independência dos processos eletroquímicos e influência dos eletrólitos PBS (pH 7,0) e $\mathrm{HCl}(\mathrm{pH} 1,0)$. Finalmente, a oxidação da cisteína, em diversas concentrações, foi avaliada utilizando-se os eletrodos ITO e ITO-(PAH/FtTsCo) ${ }_{3} \mathrm{em}$ tampão PBS (pH 7,0).

\section{RESULTADOS E DISCUSSÃO}

\section{Deposição das multicamadas PAH/FtTsCo}

Com objetivo de avaliar o estado organizacional das FtTsCo suportadas em filmes LbL, realizou-se um estudo comparativo destas
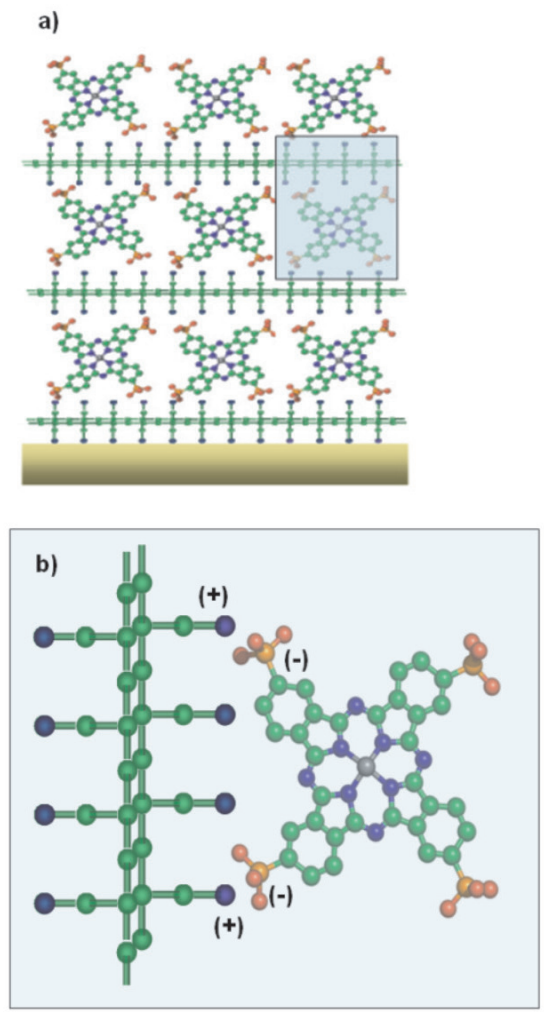

Figura 1. a) Automontagem supramolecular ilustrando a formação de 3 bicamadas de PAH/FtTsCo sobre um substrato sólido. Os átomos estão assim representados: cinza (cobalto (II)), vermelho (oxigênio), laranja (enxofre), verde (carbono) e azul (nitrogênio). Os átomos de hidrogênioforam omitidos. b) Detalhe da interação eletrostática entre as aminas protonadas do polímero (PAH) e o grupo sulfônico negativamente carregado da FtTsCo. Embora não representado no modelo, pode haver interpenetração das camadas poliméri$\operatorname{cas}^{7}$ e distorção do plano axial do metal ligante ${ }^{13}$

espécies em solução, imobilizadas em filmes automontados. Neste sentido, registraram-se os espectros eletrônicos na região do visível de uma solução contendo 7,0 x 10-6 $\mathrm{mol} \mathrm{L}^{-1}$ de FtTsCo e de um filme contendo 20 bicamadas de PAH/FtTsCo (Figura 2a). Para ambos os casos, os espectros exibem uma banda Soret (ou banda B, com maior energia) em $340 \mathrm{~nm}$, característica dos anéis porfirínicos, e uma banda Q desdobrada, devido à ocorrência de espécies diméricas e monoméricas (ombro com menor energia) na região entre 600 e $700 \mathrm{~nm} .^{12,16,18,20,21,45-}$ ${ }^{47,50}$ Cabe ressaltar que bandas do espectro eletrônico do dímero e monômero (banda Q) para o filme (PAH/FtTsCo) ${ }_{20}$ apresentaram seus máximos de absorção deslocados (618 e 692 nm), comparativamente ao espectro eletrônico da FtTsCo em solução (633 e 662 nm), bem como mais alargadas, similar ao observado previamente. ${ }^{2,9,13,50}$ Segundo Lvov, ${ }^{20}$ transições eletrônicas próximas de $630 \mathrm{~nm}$ podem também ser interpretadas em função da formação de agregados, devido ao acoplamento extensivo do sistema de elétrons $\pi$ dos anéis adjacentes. Em virtude da planaridade das moléculas e do elevado grau de conjugação eletrônica, interações associativas hidrofóbicas entre os anéis também são esperadas. ${ }^{50}$ Ressalte-se que as espécies diméricas em solução estão em maior concentração do que as espécies monoméricas para filmes construídos com até 3 bicamadas. Uma forma simples de observar essa relação é calcular as intensidades de absorção relativa entre o dímero e monômero $\left(\mathrm{I}_{\text {dim }} / \mathrm{I}_{\text {mon }}\right)$. Para FtTsCo em solução, a relação $\mathrm{I}_{633} / \mathrm{I}_{662}$ é igual a 1,5, enquanto no filme com uma bicamada de PAH/FtTsCo, a relação $\mathrm{I}_{618} / \mathrm{I}_{692}$ inicia-se em 1,2 e cresce até 5 bicamadas, quando atinge o valor de 1,7, permanecendo com o mesmo valor até 20 bicamadas. Ou seja, uma maior quantidade de dímero é favorecida em filmes LbL com maior 

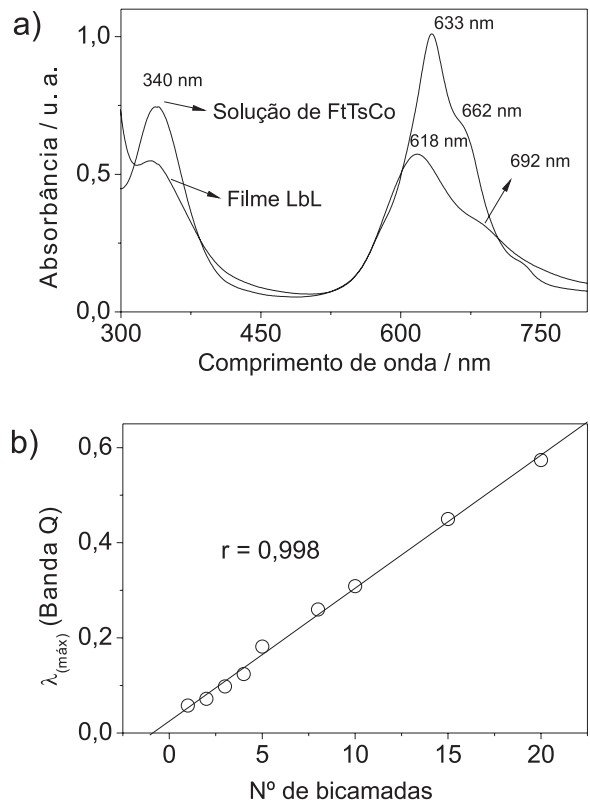

Figura 2. Espectros eletrônicos da região do visível para: a) solução diluída de FtTs Co em $\mathrm{H}_{2} \mathrm{SO}_{4}$ pH 2,5 e filme LbL (PAH/FtTsCo $)_{20}$; b) influência do número de bicamadas na absorbância da banda Q em $617 \mathrm{~nm}$

espessura (entre 5 e 20 bicamadas). Este primeiro estudo revela que a FtTsCo, em filmes LbL com até 3 bicamadas, se encontra em um estado organizacional mais espaçado, considerando a interação intermolecular FtTsCo-FtTsCo. Logo, a baixa interação entre moléculas de FtTsCo adjacentes evita as conformações do tipo $\pi-\pi$ (pi stacking). Isto pode ser atribuído a estabilização dos grupos sulfônicos das moléculas de FtTsCo pelos grupamentos amina do polieletrólito PAH no substrato, fato que pode contribuir para uma distribuição mais uniforme e diluída ao longo do filme. Como um segundo fato que leva a esta conclusão, as bandas no espectro eletrônico para o filme LbL se mostraram mais alargadas, devido às interações dos grupos iônicos. ${ }^{12,13,20}$

Outros tipos de interações também podem ocorrer em filmes LbL nanoestruturados, como a quebra de simetria no plano metal-ligante. ${ }^{13}$ Em um outro trabalho, utilizando ftalocianinas tetrassulfonadas de níquel (II), mostramos que a estabilização do complexo metálico em filmes automontados é decisivamente correlacionada à ligação do tipo ponte salina $\left(\mathrm{SO}_{3}{ }^{-}-{ }_{---{ }^{+}} \mathrm{NH}_{4}\right),{ }^{12}$ resultando em filmes extremamente estáveis. Cabe aqui frisar que a espécie PAH não apresenta transição eletrônica na região do visível, servindo apenas como um suporte polimérico para construção de multicamadas, devido ao reconhecimento molecular entre os grupos $-\mathrm{NH}_{3}^{+}(\mathrm{PAH})$ e $-\mathrm{SO}_{3}^{-}(\mathrm{FtTsCo})$. É com base neste preceito que a construção de estruturas supramoleculares multicamadas de PAH/FtTsCo se torna possível. Além disso, o crescimento da arquitetura supramolecular só é possível devido a um fenômeno conhecido como supercompensação de cargas, ${ }^{1,51}$ onde a última camada depositada, além de neutralizar a camada anterior, forma novos sítios, possibilitando a deposição de outra camada de carga oposta. ${ }^{1,51}$

Uma vez que a concentração de espécies adsorvidas em um substrato sólido é proporcional à absorbância na região do visível, monitorou-se a deposição das multicamadas de $\mathrm{PAH} / \mathrm{FtTsCo}$ por meio do crescimento da banda Q (618 nm). Na Figura 2b, observa-se que o crescimento da arquitetura supramolecular de $\mathrm{PAH} / \mathrm{FtTsCo}$ é progressivo e linear, o que acarreta em etapas de deposição uniformes. . $^{12,13,19,20,22}$ Resultados similares foram encontrados nos sistemas PAH-AuNP/ $\mathrm{FtTsNi}^{41}{ }^{11} \mathrm{PAH} / \mathrm{FtTsM},{ }^{12}$ e Quitosana/FtTsM (onde $\mathrm{M}=\mathrm{Fe}, \mathrm{Ni}$ e $\mathrm{Cu}) .{ }^{16,18}$ Conhecendo-se o estado organizacional e as propriedades de crescimento do filme, pode-se avaliar as propriedades eletroquímicas como função destes parâmetros, como mostram os resultados a seguir.

\section{Estudo eletroquímico do sistema supramolecular (PAH/ FtTsCo)}

Similar à espectroscopia eletrônica, o crescimento das multicamadas de PAH/FtTsCo foi também acompanhado utilizando-se voltametria cíclica. Porém, utilizaram-se eletrodos de ITO, cujas propriedades eletroquímicas e de interface já vêm sendo estudadas pelos autores para aplicação em filmes nanoestruturados. ${ }^{2,12,14,16-18,41}$ Antes de conhecer os efeitos da quantidade de material adsorvido em cada etapa de deposição, é preciso entender o comportamento das reações de transferência de carga entre o PAH/FtTsCo e a superfície do eletrodo. A Figura 3 mostra 20 ciclos voltamétricos utilizando um eletrodo de ITO-(PAH/FtTsCo) ${ }_{3}$, em solução de PBS pH 7,0. A utilização de um eletrodo modificado contendo apenas 3 bicamadas foi intencional pois, como veremos a seguir, a quantidade de multicamadas depositadas influencia consideravelmente os processos redox. Para o estudo eletroquímico, utilizou-se eletrólito com características fisiológicas, devido ao estudo envolvendo o aminoácido cisteína que contempla a parte final deste artigo. Utilizando essas condições, o filme PAH/FtTsCo com 3 bicamadas apresentou alta estabilidade eletroquímica, uma vez que as correntes faradaicas não se modificaram quando a janela de potencial de trabalho $(-1,0$ a $0,8 \mathrm{~V}$ vs ECS) foi ciclada durante 20 vezes em solução PBS pH 7,0 (Figura 3). Realizaramse também alguns testes em meio ácido $(\mathrm{HCl})$, com intuito de conhecer as propriedades de transferência de carga nessa situação. Diferente ao observado para os sistemas PAH/FtTsFe, ${ }^{12} \mathrm{PAH} /$ FtTsNi, ${ }^{12}$ Quitosana/FtTsNi, ${ }^{17}$ Quitosana/FtTsFe ${ }^{17}$ e Quitosana/ FtTsCu, ${ }^{2}$ o sistema (PAH/FtTsCo) não apresentou estabilidade em eletrólito de $\mathrm{HCl}$ pH 1,0. Em meio ácido, o filme (PAH/FtTsCo) mostrou-se instável, devido à diminuição das correntes anódica e catódica dos processos redox durante os sucessivos ciclos (resultado não apresentado). Segundo Lever, ${ }^{45-47}$ o fenômeno de agregação é comumente observado em estudos com ftalocianinas metálicas; porém, alguns fatores podem influenciar na formação de agregados como diminuição do $\mathrm{pH}$, aumento da concentração de ftalocianina e também da força iônica do eletrólito suporte. Em recente estudo, ${ }^{41}$ mostramos que a intercalação de PAH (ou PAHAuNP) e FtTsNi provoca uma diminuição de espécies agregadas à superfície do eletrodo, como evidenciado nos filmes PAH/FtTsNi e PAH-AuNP/FtTsNi por espectroscopia vibracional na região do infravermelho, nos quais o efeito intermolecular fônon, comum em agregados de FtTsM no estado sólido, não foi observado. ${ }^{41}$

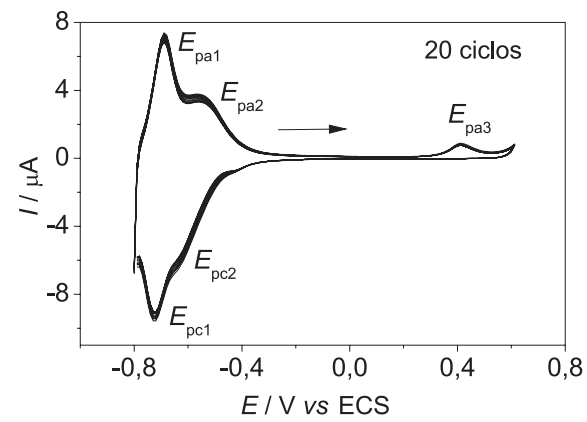

Figura 3. Voltamogramas cíclicos do filme (PAH/FtTsCo) ${ }_{3}$ com 20 ciclos em PBS 0,1 mol L $L^{-1} \mathrm{pH} \mathrm{7,0}$. Velocidade de varredura $=25 \mathrm{mV} \mathrm{s}^{-1}, \mathrm{~T}=25^{\circ} \mathrm{C}$

Na janela de potencial estudada ( $-0,8$ a $0,6 \mathrm{~V}$ vs SCE), o sistema $\mathrm{PAH} / \mathrm{FtTsCo}$ apresentou processos redox com valores de $E_{1 / 2}$ em $-0,72$ e $-0,58 \mathrm{~V}$, atribuídos aos pares redox $[\mathrm{FtTs}]^{6-} /[\mathrm{FtTs}]^{5-}$ e $[\mathrm{FtTsCo}(\mathrm{I})]^{5-} /$ 
$[\mathrm{FtTsCo}(\mathrm{II})]^{4-}$, respectivamente, e um pico em $0,40 \mathrm{~V}$ correspondente ao processo de oxidação irreversível de $[\mathrm{FtTsCo}(\mathrm{II})]^{4-}$ para $[\text { FtTsCo(III) }]^{3-}$ (Figura 3). ${ }^{20,43,44}$ Processos irreversíveis envolvendo ftalocianinas metálicas são comuns devido, principalmente, a efeitos de agregação da espécie FtTsCo(II) na superfície do eletrodo. ${ }^{20,23,45-47}$ Porém, até o presente momento, não se pode afirmar se este é o principal fator da presença do pico irreversível em $0,40 \mathrm{~V}$, uma vez que outros processos químicos após a aplicação do potencial podem ocorrer. ${ }^{52}$ As reações a seguir descrevem as etapas redox envolvidas no sistema PAH/FtTsCo:

$[\mathrm{FtTs}]^{6-} \rightarrow[\mathrm{FtTs}]^{5-}+\mathrm{e}^{-}\left(E_{\mathrm{pal}}\right)$

$[\operatorname{FtTsCo}(\mathrm{I})]^{5-} \rightarrow[\mathrm{FtTsCo}(\mathrm{II})]^{4-}+\mathrm{e}^{-}\left(E_{\mathrm{pa} 2}\right)$

$\left.[\mathrm{FtTsCo}(\mathrm{II})]^{4-} \rightarrow\left[\mathrm{FtTsCo}^{\mathrm{IIII}}\right)\right]^{3-}+\mathrm{e}^{-}\left(\mathrm{E}_{\mathrm{pa} 3}\right)$

$[\mathrm{FtTsCo}(\mathrm{II})]^{4-}+\mathrm{e}^{-} \rightarrow[\operatorname{FtTsCo}(\mathrm{I})]^{5-}\left(E_{\mathrm{pc} 2}\right)$

$[\mathrm{FtTs}]^{5-}+\mathrm{e}^{-} \rightarrow[\mathrm{FtTs}]^{6-}\left(E_{\mathrm{pcl}}\right)$

Uma vez compreendida a eletroquímica dos processos redox do sistema $\mathrm{PAH} / \mathrm{FtTsCo}$, correlacionaram-se as correntes de pico em função do número de bicamadas. Para cada etapa de deposição (1 a 20 bicamadas), os voltamogramas revelaram um aumento das correntes faradaicas (Figura 4a), sugerindo que novas multicamadas foram incorporadas, similar ao perfil observado no estudo por espectroscopia eletrônica na região do visível. Com intuito de observar as variações de corrente em função do número de bicamadas (Figura 4a), não se adotou o processo de oxidação da ftalocianina $\left(E_{\mathrm{pa1}}\right)$ para acompanhar a evolução da corrente faradaica $\left(I_{\mathrm{pa1}}\right)$ dos filmes construídos, em virtude do potencial deste pico se deslocar para potenciais menos negativos com a incorporação de novas bicamadas. A Figura $4 \mathrm{~b}$ mostra um gráfico $I_{\mathrm{pcl}}$ versus número de bicamadas, onde ocorre um crescimento linear da corrente em função do número de bicamadas.
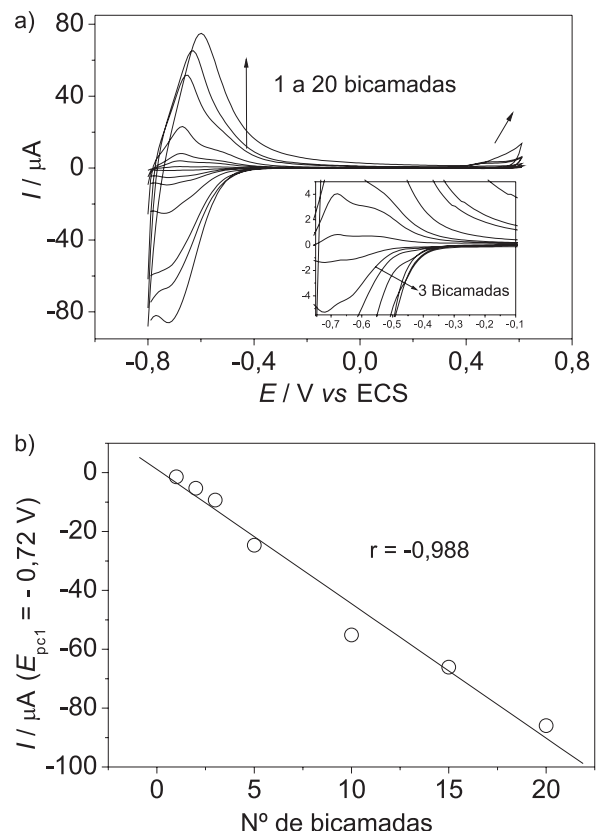

Figura 4. a) Voltamogramas cíclicos do filme PAH/FtTsCo com diferentes números de bicamadas. Eletrólito: $P B S-0,1 \mathrm{~mol} \mathrm{~L}^{-1} \mathrm{pH}$ 7,0. Velocidade de varredura $25 \mathrm{mV} \mathrm{s}^{-1}, T=25^{\circ} \mathrm{C}$. Em destaque, aumento da região catódica. b) Corrente de pico catódica $\left(I_{p c l}\right)$ versus número de bicamadas para sistema (PAH/FtTsCo)n
O sistema (PAH/FtTsCo) apresenta um deslocamento do primeiro potencial de pico anódico $\left(E_{\mathrm{pal}}\right)$ em função do aumento das multicamadas e, portanto, sobrepõe-se ao processo $E_{\mathrm{pa} 2}$ (Figura 4a). Para o processo $E_{\mathrm{pa3}}$, o deslocamento ocorre para potenciais mais positivos, de modo que no filme com 10 bicamadas o processo $E_{\text {pa } 3}$ apresenta o seu pico consideravelmente deslocado. Comportamento similar tem sido observado em outros sistemas contendo ftalocianinas; porém, em região mais anódica ( 0,0 a $0,9 \mathrm{~V}$ vs ECS $)^{12,41}$ e/ou utilizando-se eletrodos de carbono vítreo. ${ }^{25}$ Observou-se também que não há deslocamentos nos processos catódicos $E_{\mathrm{pa1}}$ e $E_{\mathrm{pa} 2}$, ocorrendo apenas a sobreposição dos picos para filmes com número de bicamadas acima de 3 . A tendência de um pico sobrepor o outro pode ser compreendida sob o ponto de vista cinético, visto que as reações mais lentas ficam prejudicadas com a distância de espécies FtTsNi ao eletrodo. Por exemplo, para ftalocianinas de níquel imobilizadas em filmes LbL PAH/FtTsNi, o primeiro processo de oxidação ocorre no ligante ftalocianina $\left(E_{\mathrm{pa1}}\right)$ e o segundo processo de oxidação, no centro $\mathrm{Ni}^{2+}\left(E_{\mathrm{pa} 2}\right) \cdot{ }^{12}$ Neste modelo, quando os voltamogramas cíclicos foram registrados em diferentes velocidades de varredura ( 5 a $500 \mathrm{mV} \mathrm{s}^{-1}$ ), observou-se que, em velocidades de varredura acima de $100 \mathrm{mV} \mathrm{s}^{-1}$, apenas o segundo processo eletroquímico $\left(E_{\mathrm{pa} 2}\right)$ apresenta uma rápida reação de transferência de carga. ${ }^{12}$

Para explicar os deslocamentos dos picos com o aumento do número de bicamadas, é preciso compreender a estrutura da interface eletrodo/eletrólito. Com o aumento do número de camadas de FtTsCo e PAH, ocorre também um aumento da quantidade de material não condutor sobre o substrato - neste caso, o polímero $\mathrm{PAH}$ pode dificultar a transferência eletrônica entre os centros redox das FtTsCo, principalmente das camadas mais distantes ao eletrodo. Assim, o sobrepotencial aplicado para oxidar ou reduzir deverá ser maior, com o intuito de romper essa barreira energética. ${ }^{52,53}$ Embora seja uma questão muito discutida na literatura, grande parte dos pesquisadores entendem que filmes automontados possuem grande permeabilidade a íons, ou seja, podem agir como membranas estruturadas que facilitam a percolação de eletrólito em seu interior causando, também, uma compensação de cargas durante os processos eletródicos. ${ }^{27}$ Existem casos, como ocorre para dendrímeros ${ }^{16,18} \mathrm{e}$ filmes de quitosana, ${ }^{17} \mathrm{em}$ que a presença do polieletrólito na estrutura não prejudica a difusão iônica e a transferência de carga ao longo de várias bicamadas (até 20 bicamadas, por exemplo). Outro ponto importante a ser considerado vai ao encontro do trabalho de Laurent e Shelenoff, ${ }^{53}$ os quais propuseram que a transferência de elétrons em filmes automontados contendo moléculas redox ocorre via electron hopping, mecanismo que ocorre entre os sítios redox vizinhos ao longo de toda a extensão do filme. Estes pesquisadores ${ }^{53}$ partiram do princípio de que o tunelamento dos elétrons para a superfície do eletrodo só pode ocorrer a poucos ângstrons de distância do centro redox e que um potencial químico surge ao longo das multicamadas. Geralmente, em filmes automontados contendo PAH, as bicamadas podem ter cerca de dezenas de ângstrons. ${ }^{2,13}$ Para filmes automontados contendo ftalocianinas metálicas, o mecanismo de electron hopping é o mais provável e isso tem sido revelado em trabalhos recentes..$^{2,12,13,16-18,41}$ No caso das multicamadas de PAH/FtTsCo, uma possível explicação para a perda de reversibilidade eletroquímica dos pares redox e para os deslocamentos de potencial pode estar associada a alto grau de empacotamento molecular com o aumento do número de bicamadas. Neste caso, o embasamento está no fato de que moléculas empacotadas tendem a se aglomerar e, por isso, a ocorrência de dímeros nestas arquiteturas supramoleculares com filmes mais espessos é evidenciada.

\section{Cinética de transferência de carga}

Nos voltamogramas da Figura 5, analisou-se o transporte de carga nas multicamadas por meio da influência das velocidades de 
varredura (10 até $\left.500 \mathrm{mV} \mathrm{s}^{-1}\right)$ em diferentes arquiteturas supramoleculares (números de bicamadas). Para o sistema PAH/FtTsCo, as correntes de pico aumentaram linearmente até a velocidade de 500 $\mathrm{mV} \mathrm{s}^{-1}$, indicando um mecanismo com rápida transferência de carga entre as multicamadas. ${ }^{52,53}$ Como exemplo, para o filme LbL com 3 bicamadas (Figura 6), os picos das correntes anódica $\left(I_{\mathrm{pal}}\right)$ e catódica $\left(I_{\mathrm{pcl}}\right)$ aumentam linearmente em função da velocidade de varredura, indicando corrente limitada pela transferência de carga. ${ }^{17}$ Embora alguns autores mencionem que existam processos limitados por difusão em filmes automontados com ftalocianinas imobilizadas, ${ }^{13,20}$ acredita-se que esse mecanismo não pode ocorrer, devido às próprias características desse sistema. Por exemplo, quando um potencial é aplicado ao eletrodo de trabalho contendo os filmes com FtTsCo, a quantidade de material eletroativo na camada de difusão não varia pois, em princípio, não há movimentação dessas espécies na interface. Esse fato é corroborado pela ligação estável formada entre os grupamentos sulfônicos e aminas que originam os filmes multicamadas, como discutido anteriormente. Para compensar a carga gerada durante a aplicação do potencial, ocorre a movimentação de íons no sentido contrário, levando ao balanço de corrente faradaica obtida no sistema. Caso não haja uma expressiva permeação de eletrólito no filme, os processos redox envolvendo as moléculas de FtTsCo podem ficar limitados pela migração dos íons. Porém, não acreditamos que isso possa ocorrer, pois, como demonstrado por vários autores, ${ }^{2,12,41,53}$ uma das vantagens em se utilizar polieletrólitos em eletrodos é sua alta permeabilidade aos íons. Além disso, o eletrólito suporte se encontra em altas concentrações $\left(0,1 \mathrm{~mol} \mathrm{~L}^{-1}\right)$, o que facilita a sua difusão. Outro fator importante a ser comentado é que a aplicação de filmes automontados como barreira para a difusão de íons (membranas seletivas) só ocorre, geralmente, quando a última camada de polieletrólito atua na repulsão eletrostática de íons, consequência do processo de supercompensação de cargas. ${ }^{54}$ Porém, os íons geralmente estão em baixas concentrações e tal mecanismo é utilizado para eliminar efeitos de interferentes em solução. ${ }^{54,55}$

No sistema (PAH/FtTsCo) observa-se que o processo de oxidação da ftalocianina $\left(E_{\mathrm{pal}}\right)$ se desloca para potenciais mais positivos quando há um aumento da velocidade de varredura, sobrepondo o primeiro processo de oxidação do cobalto $\left(E_{\mathrm{pa} 2}\right)$, sugerindo irreversibilidade ao sistema (Figura 5). ${ }^{20} \mathrm{~A}$ componente $E_{\mathrm{pa} 3}$ também se desloca para potenciais mais positivos quando há um aumento da velocidade de varredura nas diferentes bicamadas, indicando um caráter irreversível. Este último indício fica mais acentuado nos filmes multicamadas de PAH e FtTsCo com 5 e 10 bicamadas (Figuras 5c e 5d). Além disso, a componente catódica $\left(E_{\mathrm{pc} 3}\right)$ não foi observada no sistema multicamadas com FtTsCo, nem mesmo em baixas velocidades de varredura $\left(5 \mathrm{mV} \mathrm{s}^{-1}\right)$. O eletrodo modificado ITO-(PAH/FtTsCo) ${ }_{3}$ apresentou dois processos redox com valores de $E_{(1 / 2) 1}$ e $E_{(1 / 2) 2}$ em - 0,72 e - 0,58 $\mathrm{V}$, respectivamente, e um $E_{\mathrm{pa} 3}$ em $0,40 \mathrm{~V}$. Observou-se que a resposta eletroquímica é proporcional ao número de sítios ativos redox acessíveis da FtTsCo e que o substrato de ITO não apresenta resposta eletroquímica na faixa de potencial estudada (MS). ${ }^{14} \mathrm{~A}$ interpretação de cada etapa eletroquímica é muito importante pois, como veremos a seguir, a atividade catalítica depende significativamente de cada processo envolvido.

\section{Efeito supramolecular e atividade eletrocatalítica do eletrodo de ITO-(PAH/FtTsCo), para oxidar cisteína}

Para avaliar a atividade eletrocatalítica do filme de PAH/FtTsCo, escolheu-se o aminoácido cisteína para nossos estudos. O motivo dessa escolha vem do fato da literatura ${ }^{26,56,57}$ reportar vários mecanismos para eletroxidação de cisteína pela FtTsCo. Assim, pode-se avaliar o efeito da estruturação do filme de $\mathrm{PAH} / \mathrm{FtTsCo.}$
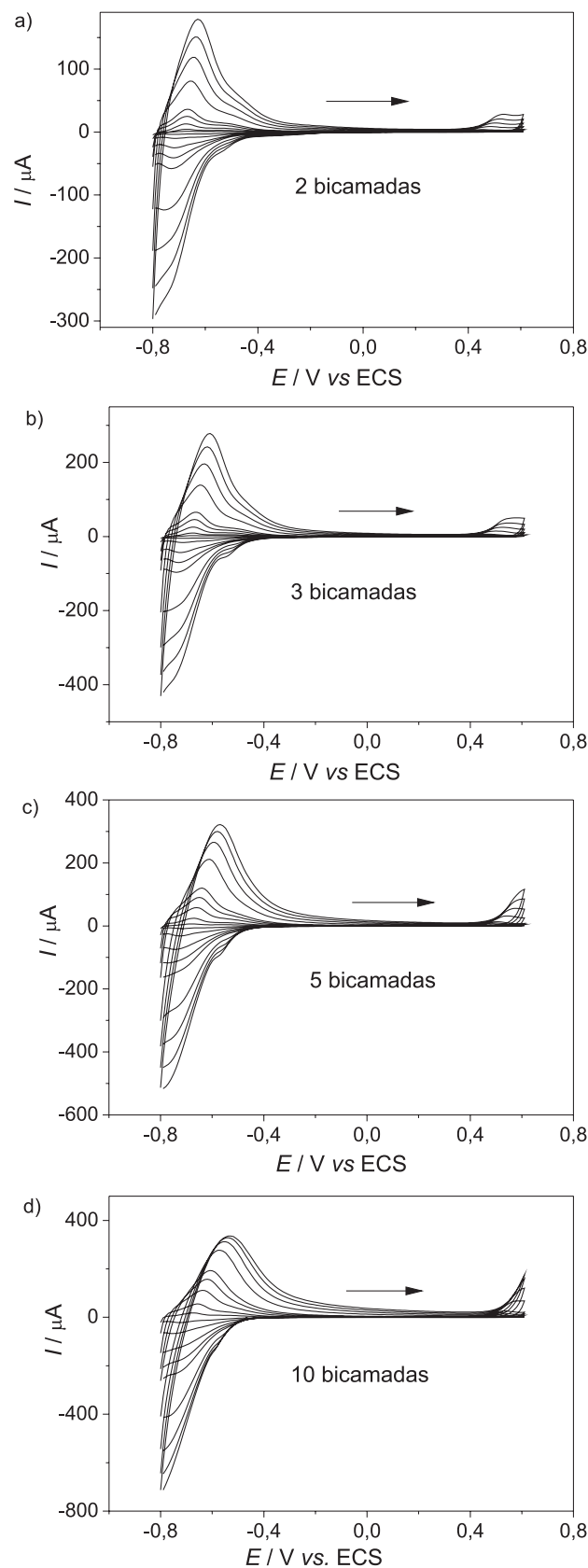

Figura 5. Voltamogramas cíclicos do filme LbL PAH/FtTsCo com: a) 2, b) 3 , c) 5 e d) 10 bicamadas em diferentes velocidades $(25,75,200,300,400 e$ $500 \mathrm{mV} \mathrm{s}^{-1}$ ). Eletrólito: $P B S-0,1 \mathrm{~mol} \mathrm{~L} L^{-1} \mathrm{pH} \mathrm{7,0,T}=25^{\circ} \mathrm{C}$

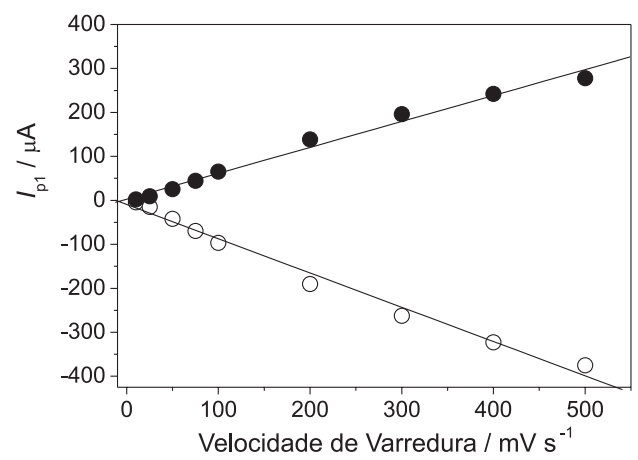

Figura 6. Incremento da corrente de pico anódica e catódica em função do aumento da velocidade de varredura, utilizando um eletrodo de ITO com 3 bicamadas de PAH/FtTsCo 
Além disso, estudos envolvendo aminoácidos e sistemas nanoestruturados têm se tornado um grande desafio na compreensão dos efeitos biomoleculares em nanoescala. ${ }^{58}$ Mais especificamente, moléculas que apresentam o grupamento sulfidrila (-SH), como cisteína e glutationa, têm se destacado devido à relevância no meio intracelular. ${ }^{22-25,48}$

Segundo Cookson e colaboradores, ${ }^{56}$ a interação entre FtTsCo(II) e a cisteína resulta na formação da espécie FtTsCo(I) e cistina, sendo que a regeneração da espécie FtTsCo(II), na presença de cisteína, ocorre somente em pHs maiores que 4,0. De acordo com Kubota e colaboradores, ${ }^{57}$ em filmes de FtTsCo/PLL (PLL = poli-lisina) a eletroxidação da cisteína, para cistina por espécies FtTsCo(II), ocorre segundo um mecanismo em quatro etapas:

$\mathrm{RSH}+\mathrm{OH}^{-} \rightarrow \mathrm{RS}^{-}+\mathrm{H}_{2} \mathrm{O}$

$[\mathrm{FtTsCo}(\mathrm{II})]^{2-}+\mathrm{RS}^{-} \rightarrow\left[\mathrm{FtTsCo}(\mathrm{I}) \mathrm{RS}^{2-}\right.$

$[\mathrm{FtTsCo}(\mathrm{I}) \mathrm{RS}]^{2-} \rightarrow[\mathrm{FtTsCo}(\mathrm{I})]^{3-}+\mathrm{RS}^{\cdot+}$

$[\mathrm{FtTsCo}(\mathrm{I})]^{3-} \rightarrow[\mathrm{FtTsCo}(\mathrm{II})]^{2-}+\mathrm{e}-$

onde, $\mathrm{RSH}=$ cisteína e $\mathrm{RS}^{\cdot+}$ = cátion radical da cisteína .

Sekota e Nyokong ${ }^{26}$ também propõem um mecanismo de catalisador reversível, onde as espécies FtTsCo(III) são responsáveis pela eletroxidação da cisteína, com formação de cistina e FtTsCo(II), segundo as equações:

$[\mathrm{FtTsCo}(11)]^{4-} \rightarrow[\mathrm{FtTsCo}(\mathrm{III})]^{3-}+\mathrm{e}^{-}$

$[\mathrm{FtTsCo}(\mathrm{III})]^{3-}+\mathrm{RSH} \rightarrow[\mathrm{FtTsCo}(11)]^{4-}+\mathrm{RS}^{\bullet}+\mathrm{H}^{+}$

$2 \mathrm{RS} \cdot \rightarrow \mathrm{RSSR}$

Diante dos vários modelos propostos, algumas perguntas ainda são pertinentes: Qual espécie química é responsável pela eletrocatálise da cisteína em nível supramolecular? Podem, realmente, compostos de FtTsCo atuar como mediadores reversíveis de elétrons? Existe a possibilidade da formação de um intermediário (ex. complexo cisteína-FtTsCo) que facilite a oxidação da cisteína? Para tentar responder essas perguntas, elaborou-se uma estratégia onde comparamos diferentes sistemas contendo FtTsCo na modificação eletródica.

No primeiro sistema, adsorveu-se ftalocianinas de cobalto em eletrodo de ITO por meio de sucessivas ciclagens do mesmo em solução contendo FtTsCo em meio ácido $(\mathrm{pH} 1,0) .{ }^{12}$ Vale a pena mencionar que as ftalocianinas metálicas apresentam processos de eletropolimerização em valores acima de $\mathrm{pH} 7,0,{ }^{24}$ fato que motivou o estudo do comportamento da FtTsCo após imobilização em meio ácido. Para adsorção da FtTsCo, obtiveram-se vários ciclos voltamétricos (janela de potencial $=-0,8$ a 0,6 V, 50 ciclos) utilizando ITO como eletrodo de trabalho, em uma solução de 0,5 $\mathrm{g} \mathrm{L}^{-1}$ de FtTsCo em meio de $\mathrm{HCl} \mathrm{0,1} \mathrm{mol} \mathrm{L}^{-1}$. Após a adsorção da FtTsCo, o eletrodo modificado (ITO-FtTsCo) foi lavado para eliminar o excesso de espécies que não foram adsorvidas e, em seguida, seco em gás nitrogênio. Os voltamogramas cíclicos com diferentes velocidades de varredura para as espécies FtTsCo adsorvidas em ITO, utilizando PBS ( $\mathrm{pH} 7,0$ ) como eletrólito, apresentaram um par redox com valor de $E_{1 / 2}$ em - 0,23 V (vs SCE) e uma curva com o perfil típico de espécies reversíveis adsorvidas (MS). Além disso, este eletrodo apresentou alta resistividade e maior corrente capacitiva. O perfil apresentado pelo voltamograma cíclico é muito semelhante quando comparado com outros sistemas relatados na literatura, ${ }^{14,20,57}$ mesmo que o processo de fabricação dos eletrodos modificados não seja o mesmo. Comparando os voltamogramas dos sistemas ITO-FtTsCo e ITO-(PAH/FtTsCo) ${ }_{3}$, em uma escala com corrente normalizada (MS), observa-se claramente que o processo envolvido está relacionado a uma possível sobreposição das etapas de conversão: $[\mathrm{FtTsCo}(\mathrm{I})]^{5-} \rightarrow[\mathrm{FtTsCo}(\mathrm{II})]^{4-} \mathrm{e}$ $\left.\left[\mathrm{FtTsCo}_{\mathrm{II}}\right]^{4-} \rightarrow\left[\mathrm{FtTsCo}^{\mathrm{III}}\right)\right]^{3-}$. Assim, constatou-se que os processos redox na região entre $-0,8$ e $-0,5$, observados nos filmes LbL, se evidenciam apenas quando as moléculas de FtTsCo estão estruturadas em ambiente supramolecular na superfície eletródica. Neste sentido, utilizou-se o eletrodo de ITO-(PAH/FtTsCo) ${ }_{3}$ na oxidação da cisteína. Na Figura 7, observam-se os voltamogramas do eletrodo ITO-(PAH/FtTsCo) ${ }_{3}$ em meio PBS $0,1 \mathrm{~mol} \mathrm{~L}^{-1}$, com velocidade de varredura de $25 \mathrm{mV} \mathrm{s}^{-1}$, para adição de cisteína na faixa de concentração de $1,0 \times 10^{-4}$ a $1,6 \times 10^{-3} \mathrm{~mol} \mathrm{~L}^{-1}$. Neste sistema, há um aumento na corrente anódica (a partir de $0,4 \mathrm{~V}$ ) em função da concentração da cisteína, bem como um pequeno aumento das correntes dos processos eletroquímicos em região catódica. Comparando a atividade eletrocatalítica dos sistemas ITO puro e ITO modificado com filme (MS), na mesma concentração de cisteína $\left(1,6 \times 10^{-3} \mathrm{~mol} \mathrm{~L}^{-1}\right)$, observa-se que o sistema supramolecular ITO-(PAH/FtTsCo) ${ }_{3}$ apresenta um ganho de corrente de oxidação da cisteína em 25 vezes. Além disso, a possibilidade de coordenação da cisteína ao plano axial da FtTsCo pode ser descartada, devido à forte interação supramolecular que ocorre entre as multicamadas do polímero e do complexo metálico..$^{2,12,41}$ Mesmo com FtTsCo em solução (PBS pH 7,0), não se observou modificação no espectro eletrônico na região do UV-VIS quando se variou a concentração de cisteína em relação a FtTsCo em até 50 vezes (resultados não apresentados).

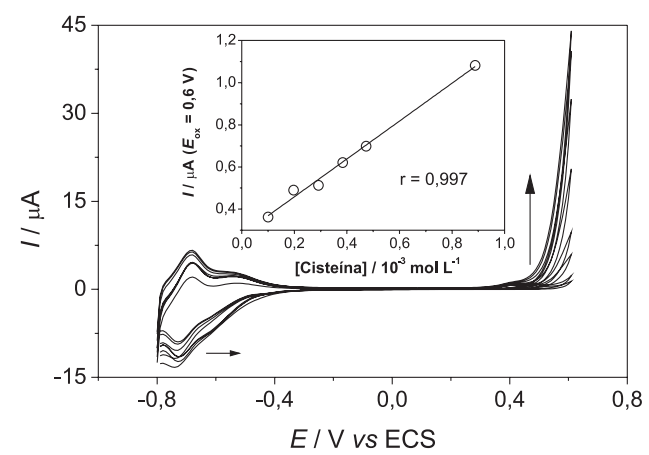

Figura 7. Voltamogramas cíclicos para filme (PAH/FtTs Co $)_{3}$ com a variação da concentração de cisteína entre $1,0 \times 10^{-4}$ e $1,6 \times 10^{-3} \mathrm{~mol} \mathrm{~L}^{-1}$ (Detalhe: relação da corrente anódica no $E_{p a}=0,6 \mathrm{~V}$ em função de concentração de cisteína). Eletrólito PBS - 0,1 mol $L^{-1} \mathrm{pH} \mathrm{7,0;} \mathrm{velocidade} \mathrm{de} \mathrm{varredura}=25$ $m V s^{-1} e T=25^{\circ} \mathrm{C}$

Com base nestas evidências, várias informações podem ser obtidas e as respostas das questões iniciais começam a ser elucidadas. $\mathrm{O}$ aumento da corrente de pico em $0,6 \mathrm{~V}$ sugere que o processo eletrocatalítico para a oxidação da cisteína ocorre, primeiramente, com a oxidação do centro metálico, $[\mathrm{FtTsCo}(\mathrm{II})]^{4-} \rightarrow[\mathrm{FtTsCo}(\mathrm{III})]^{3-}$, seguida da oxidação da cisteína. Essa pode ser a maior contribuição da FtTsCo na eletrocatálise, devido ao elevado ganho na corrente faradaica. Ademais, o efeito eletrocatalítico com o eletrodo de ITO(PAH/FtTsCo) ${ }_{3}$ é verificado pelo ganho de corrente em potenciais mais baixos, quando comparado ao ITO não modificado, cuja oxidação da cisteína só começa a efetivamente ocorrer em potenciais acima de 0,4 V. As reações 13 e 14 resumem essas observações:

$\left.\left.[\mathrm{FtTsCo}(\mathrm{II})]^{4-} \rightarrow\right] \mathrm{FtTsCo}(\mathrm{III})\right]^{3-}+\mathrm{e}^{-}$ 


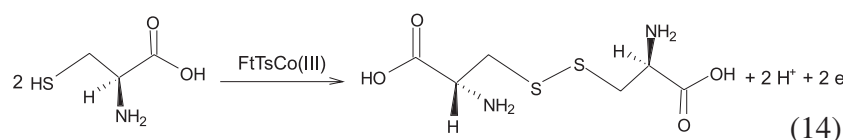

Inicialmente, observa-se neste modelo proposto a oxidação de $[\mathrm{FtTsCo}(\mathrm{II})]^{4-}$ a $[\mathrm{FtTsCo}(\mathrm{III})]^{3-}$ que, em seguida, atua na oxidação catalítica da cisteína a cistina (14). Como não há intermediários ligados ao centro metálico e a formação de complexos está descartada, acredita-se que este seja um mecanismo de esfera externa, seguido do mecanismo electron hopping. A Figura 8 ilustra todas as etapas da reação de oxidação da cisteína no eletrodo de ITO-(PAH/FtTsCo).

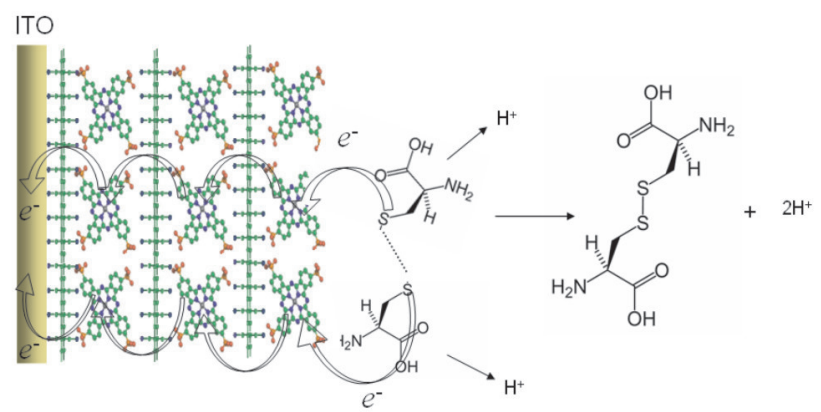

Figura 8. Esquema ilustrativo da oxidação da cisteína em um eletrodo contendo 3 bicamadas de PAH/FtTsCo(III). Primeiramente, duas moléculas de cisteína são oxidadas na superfície da FtTsCo(III) que, pelo mecanismo electron hopping, transporta os elétrons até a superfície eletródica. Durante a oxidação via reação de esfera externa, ocorre a formação de dois prótons e o transporte de dois elétrons.

\section{CONCLUSÃO}

Arranjos supramoleculares contendo o polieletrólito PAH e moléculas de FtTsCo foram estudados em filmes automontados, onde se verificou um crescimento da arquitetura supramolecular, perpendicularmente ao plano do substrato (ITO e vidro), com caráter progressivo e uniforme. Observou-se que as bandas na região do visível para o sistema PAH/FtTsCo com 3 bicamadas são mais alargadas, devido às interações intermoleculares entre os grupos $\mathrm{NH}_{3}^{+}(\mathrm{PAH})$ e $\mathrm{SO}_{3}^{-}(\mathrm{FtTsCo})$ em nível supramolecular. Nesta arquitetura, as moléculas de FtTsCo se encontram mais espaçadas no plano axial, evitando a formação de agregados e acoplamentos do tipo $\pi-\pi$. Constatou-se que a arquitetura supramolecular (número de camadas) influencia o transporte de carga entre os sítios redox da FtTsCo, uma vez que o mecanismo de electron hopping foi aplicado para justificar o aumento das correntes faradaicas nos estudos voltamétricos. Cada processo redox da FtTsCo exibe um comportamento cinético diferenciado, que pode variar com o aumento do número de bicamadas. O filme de $\mathrm{PAH} / \mathrm{FtTsCo}$ com 3 bicamadas apresentou elevada estabilidade eletroquímica em eletrólito PBS pH 7,0, o que possibilitou a comparação desse sistema com um filme de FtTsCo adsorvido eletroquimicamente, onde apenas um processo redox na espécie foi observado para esse último. Neste caso, há uma sobreposição dos picos e elevada corrente capacitiva residual. Estes resultados foram importantes para entender como ocorre o processo de oxidação catalítica do aminoácido cisteína em nível supramolecular, para o qual foi proposto um modelo envolvendo a participação da espécie $[\mathrm{FtTsCo}(\mathrm{III})]^{3-}$. Acredita-se que tais resultados credenciam a utilização do sistema supramolecular do tipo PAH/FtTsCo em áreas como catálise heterogênea, sensor e biossensor, nas quais as propriedades catalíticas e estruturais podem ser otimizadas de acordo com a arquitetura molecular.

\section{MATERIAL SUPLEMENTAR}

Disponível em http://quimicanova.sbq.org.br, na forma de arquivo PDF, com acesso livre. Figura 1S: Comparação entre os voltamogramas cíclicos dos eletrodos ITO-(PAH/FtTsCo) ${ }_{3}$ e ITO puro; Figura 2S: Voltametria cíclica utilizando um eletrodo modificado de ITOFtTsCo a partir de uma solução de $0,5 \mathrm{~g} \mathrm{~L}^{-1}$ de FtTsCo em $\mathrm{H}_{2} \mathrm{SO}_{4} \mathrm{pH}$ 2,5; Figura 3S: Voltamogramas para os sistemas ITO-FtTsCo e ITO(PAH/FtTsCo) ${ }_{3}$ com corrente normalizada e Figura 4S: Performance eletrocatalítica do ITO puro e ITO-(PAH/FtTsCo) ${ }_{3}$ na presença de cisteína em $1,6 \times 10^{-3} \mathrm{~mol} \mathrm{~L}^{-1}$.

\section{AGRADECIMENTOS}

À FAPEPI, CAPES, FAPESP e ao CNPq pelo auxílio financeiro. Os autores agradecem também ao Prof. A. C. Alves (UFPI) pela gentil revisão deste manuscrito.

\section{REFERÊNCIAS}

1. Paterno, L. G. Mattoso, L. H. C.; Oliveira Jr., O. N.; Quim. Nova 2001, 24, 228.

2. Crespilho, F. N.; Silva, W. C.; Zucolotto, V. Em Catalysis and Photochemistry in Heterogeneous Media; Nantes, I. L.; Brochsztain, S., eds.; Research Signpost: India, 2007, vol. 1, cap. 3.

3. Vidotti, M.; Silva, M. R.; Salvador, R. P.; Torresi, S. I. C.; Dall'Antonia, L. H.; Electrochim. Acta 2008, 53, 4030.

4. Volpati, D.; Alessio, P.; Zanfolim, A. A.; Storti, F. C.; Job, A. E.; Ferreira, M.; Riul Jr., A.; Oliveira Jr., O. N.; Constantino, C. J. L.; J. Phys. Chem. B 2008, 112, 15275. J.

5. Watanabe. S.; Kimura, H.; Sato, T.; Shibata, H.; Sakamoto, F.; Azumi, R.; Sakai, H.; Abe, M.; Matsumoto, M.; Langmuir 2008, 24, 8735.

6. Mandal, P.; Srinivasa, R. S.; Talwar, S. S. ; Major, S. S.; Appl. Surf. Sci. 2008, 254, 5028.

7. Decher. G.; Science 1997, 277, 1232.

8. Decher, G.; Hong, J. D.; Makromol.Chem. Macromol. Symp. 1991, 46, 321.

9. Snow, A. W.; Barger, W. R. Em Phthalocyanines - Properties and Applications; Leznoff, C. C.; Lever, A. B. P., eds.; VCH Publishers: New York, 1989, vol. 1, cap. 5.

10. Kotov, N. A.; NanoStruct. Mater. 1999, 12, 789.

11. Doherty III, W. J.; Friedlein, R.; Salaneck, W. R.; J. Phys. Chem. C 2007, 111, 2724.

12. Alencar, W. S.; Crespilho, F. N.; Santos, M. R. M. C.; Zucolotto, V.; Oliveira Jr., O. N.; Silva, W. C.; J. Phys. Chem. C 2007, 111, 12817.

13. Zucolotto, V.; Ferreira, M.; Cordeiro, M. R.; Constantino, C. J. L.; Balogh, D. T.; Zanatta, A. R.; Moreira, W. C.; Oliveira Jr., O. N.; J. Phys. Chem. B 2003, 107, 3733.

14. Crespilho, F. N.; Zucolotto, V.; Siqueira Jr., J. R.; Constantino, C. J. L.; Nart, F. C.; Oliveira Jr., O. N.; Environ. Sci. Technol. 2005, 39, 5385.

15. Zhao, S.; Li, X.; Yang, M.; Sun, C.; J. Mater. Chem. 2004, 14, 840.

16. Crespilho, F. N.; Zucolotto, V.; Brett, C. M. A.; Oliveira Jr., O. N.; Nart, F. C.; J. Phys. Chem. B 2006, 110, 17478.

17. Crespilho, F. N.; Zucolotto, V.; Siqueira Jr., J. R.; Carvalho, A. J. F.; Nart, F. C.; Oliveira Jr., O. N.; Int. J. Electrochem. Sci. 2006, 1, 151.

18. Siqueira Jr., J. R.; Crespilho, F. N.; Zucolotto, V.; Oliveira Jr., O. N.; Electrochem. Commun. 2007, 9, 2676.

19. Ferreyra, N.; Coche-Guérente, L.; Fatisson, J.; Teijelo, M. L.; Labbé, P.; Chem. Commun. 2003, 2056.

20. Lvov, Y. M.; Kamau, G. N.; Zhou, D. L.; Rusling, J. F.; J. Colloid Interface Sci. 1999, 212, 570. 
21. Zilbermann, I.; Hayon, J.; Katchalski, T.; Ydgar, R.; Rishpon, J.; Shames, A. I.; Korin, E.; Bettelheim, A.; Inorg. Chim. Acta 2000, 305, 53.

22. O'Shea, T. J.; Lunte, S. M.; Anal. Chem. 1994, 66, 307.

23. Kou, X.; Zhang, S.; Yang, Z.; Tsung, C.; Stucky, G. D.; Sun, L.; Wang, J.; Yan, C.; J. Am. Chem. Soc. 2007, 129, 6402.

24. Maree, S.; Nyokong, T.; J. Electroanal. Chem. 2000, 492, 120.

25. Luz, R. C. S.; Moreira, A. B.; Damosa, F. S.; Tanaka, A. A.; Kubota, L. T.; J. Pharm. Biom. Anal. 2006, 42, 184.

26. Sekota, M.; Nyokong, T.; Polyhedron 1997, 16, 3279.

27. Ozoemena, K.; Westbroek, P.; Nyokong, T.; Electrochem. Commun. 2001, 3, 529 .

28. Nyokong, T.; Vilakazi, S.; Talanta 2003, 61, 27.

29. Peeters, K.; Wael, K.; Bogaert, D.; Adriaens, A.; Sens. Actuators, B 2008, 128, 494.

30. Barrera, C.; Zhukov, I.; Villagra, E.; Bedioui, F.; Páez, M. A.; Costamagna, J.; Zagal, J. H.; J. Electroanal. Chem. 2006, 589, 212.

31. Sun, C.; Sun, Y.; Zhang, X.; Zhang, X.; Jiang, D.; Gao, Q.; Xu, H.; Shen, J.; Thin Solid Films 1996, 288, 291

32. Araki, K.; Toma, H. E.; Quim. Nova 2002, 25, 962.

33. Lipskier, J. F.; Tran-Thi, T. H.; Inorg. Chem. 1993, 32, 722.

34. Toma, H. E.; J. Braz. Chem. Soc. 2003, 14, 845.

35. Baioni, A. P.; Vidotti, M.; Fiorito, P. A.; Torresi, S. I. C.; J. Electroanal. Chem. 2008, 622, 219.

36. Alves, W. A.; Pfaffen, V.; Ortiz, P. I.; Torresi, S. I. C.; Torresi, R. M.; J. Braz. Chem. Soc. 2008, 19, 651.

37. Lehn, J. M.; Supramolecular Chemistry - Scope and Perspectives Molecules - Supermolecules - Molecular Devices, Nobel Lecture: Paris, 1987.

38. Lehn, J. M.; Rep. Prog. Phys. 2004, 67, 249.

39. Lehmann, P.; Symietz, C.; Brezesinski, G.; Krab, H.; Kurtth, D. G.; Langmuir 2005, 25, 5901

40. Lehn, J. M.; Chem. Soc. Rev. 2007, 36, 151.
41. Alencar, W. S.; Crespilho, F. N.; Martins, M. V. A.; Zucolotto, V.; Oliveira Jr., O. N.; Silva, W. C.; Phys. Chem. Chem. Phys. 2009, 11, 5086.

42. Zilbermann, I.; Hayon, J.; Katchalski, T.; Ydgar, R.; Rishpon, J.; Shames, A. I.; Korin, E.; Bettelheim, A.; Inorg. Chim. Acta 2000, 305, 53.

43. De Wael, K.; Westbroek, P.; Temmerman, E.; J. Electroanal. Chem. 2004, 567,167

44. Danica, M.; Fethi, B.; José, H. Z.; Electrochim. Acta 2002, 48, 323.

45. Zecevic, S.; Simic-Glavaski, B.; Yeager, E.; Lever, A. B. P.; Minor, P. C.; J. Electroanal. Chem. 1985, 196, 339.

46. Lever, A. B. P.; Henpstead, M. R.; Leznoff, C. C.; Liu, W.; Melnik, M.; Nevin, W. A.; Seymour, P.; Pure Appl. Chem. 1986, 58, 1467.

47. Lever, A. B. P.; Milaeva, E. R.; Speier, G. Em ref. 9, vol. 3, cap. 1.

48. Filho, J. R. O.; Silva, W. C.; Pereira, J. C. M.; Franco, D. W.; Inorg. Chim. Acta 2006, 359, 2888.

49. Purvis, K. L.; Lu, G.; Schwartz, J.; Bernasek, S. L.; J. Am. Chem. Soc. 2000, 122, 1808 .

50. Toma, H. E.; Silva, L.; Quim. Nova 2005, 28, 897.

51. Lösche, M.; Schimitt, J.; Decher, G.; Bouwman, W. G.; Kjaer, K.; Macromolecules 1998, 32, 8893 .

52. Brett, C. M. A.; Brett, A. M. O.; Electrochemistry: Principles, Methods and Applications, Oxford University Press: Oxford, 1993, vol. 1.

53. Laurent, D.; Schlenoff, J. B.; Langmuir 1997, 13, 1552.

54. Crespilho, F. N.; Sumodjo, P. T. A.; Esteves, M.; Podlaha, E.; Zucolotto, V.; J. Phys. Chem. C 2009, 1133, 6037.

55. Hammonds, P. T.; Adv. Mater. 2004, 16, 1271.

56. Cookson, D. J.; Smith, T. D.; Boas, J. F.; Hicks, P. R.; Pilbrow, J. R.; Chem. Soc., Dalton Trans. 1977, 109, 109.

57. Luz, R. C. S.; Moreira, A. B.; Damos, F. S.; Tanaka, A. A.; Kubota, L. T.; J. Pharm. Biomed. Anal. 2006, 42, 184.

58. Crespilho, F. N.; Lima, F. C. A.; Silva, A. B. F.; Oliveira Jr., O. N.; Zucolotto, V.; Chem. Phys. Lett. 2009, 469, 186. 


\section{ORGANIZAÇÃO SUPRAMOLECULAR DA FTALOCIANINA DE COBALTO(II) E SEU EFEITO NA OXIDAÇÃO DO AMINOÁCIDO CISTEÍNA}

Amanda Costa Santos, Roberto A. Sousa Luz, Luana G. França Ferreira, José Ribeiro dos Santos Júnior e Welter Cantanhêde da Silva*

Departamento de Química, Centro de Ciências da Natureza, Universidade Federal do Piauí, 64049-550 Teresina - PI, Brasil Frank Nelson Crespilho

Centro de Ciências Naturais e Humanas, Universidade Federal do ABC, 09210-170 Santo André - SP, Brasil

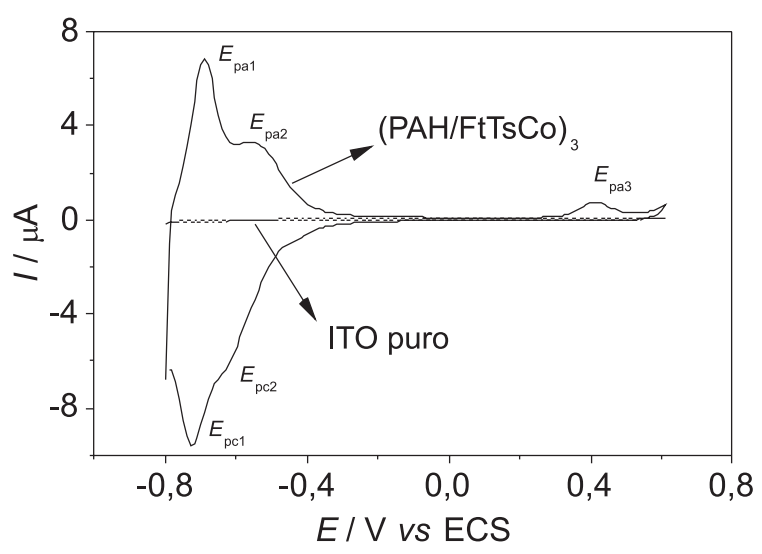

Figura 1S. Comparação entre os voltamogramas cíclicos dos eletrodos ITO(PAH/FtTs Co) ${ }_{3}$ (linha contínua) e ITO puro (linha tracejada) em PBS - 0,1 mol L $L^{-1} \mathrm{pH}$ 7,0. Dados: velocidade de varredura $=25 \mathrm{mV} \mathrm{s} \mathrm{s}^{-1}$ e T $=25^{\circ} \mathrm{C}$

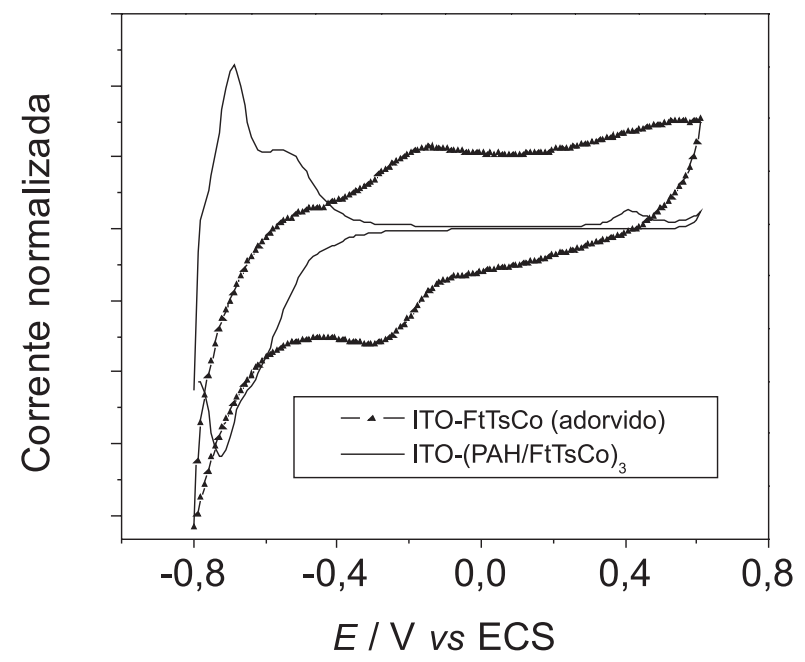

Figura 3S. Voltamogramas para os sistemas ITO-FtTsCo e ITO-(PAH/FtTs$\mathrm{Co}_{3}$, com corrente normalizada

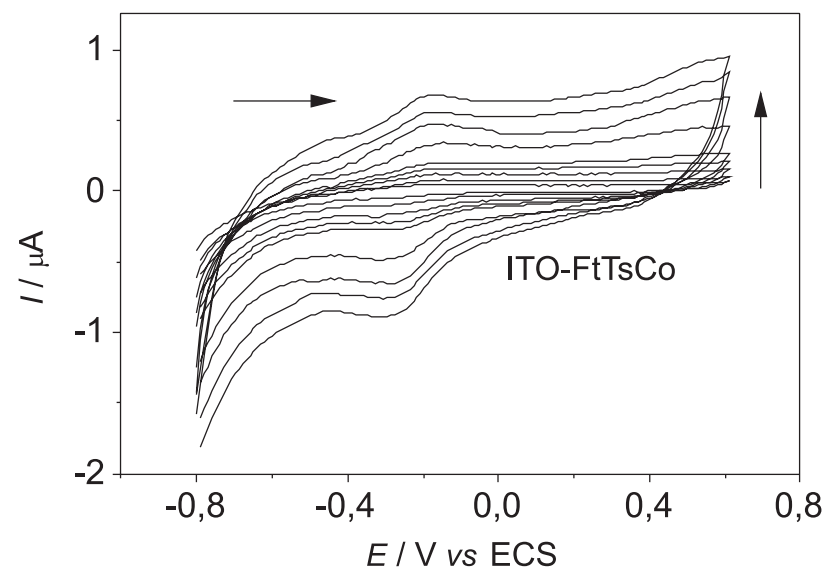

Figura 2S. Voltametria cíclica utilizando um eletrodo modificado de ITOFtTsCo a partir de uma solução de 0,5 $\mathrm{g} \mathrm{L}^{-1}$ de FtTs Co em $\mathrm{H}_{2} \mathrm{SO}_{4} \mathrm{pH}$ 2,5. Eletrólito: PBS 0,1 mol L $\mathrm{L}^{-1} \mathrm{pH}$ 7,0, velocidades de varredura: 10 - $500 \mathrm{mV} \mathrm{s}^{-1}$

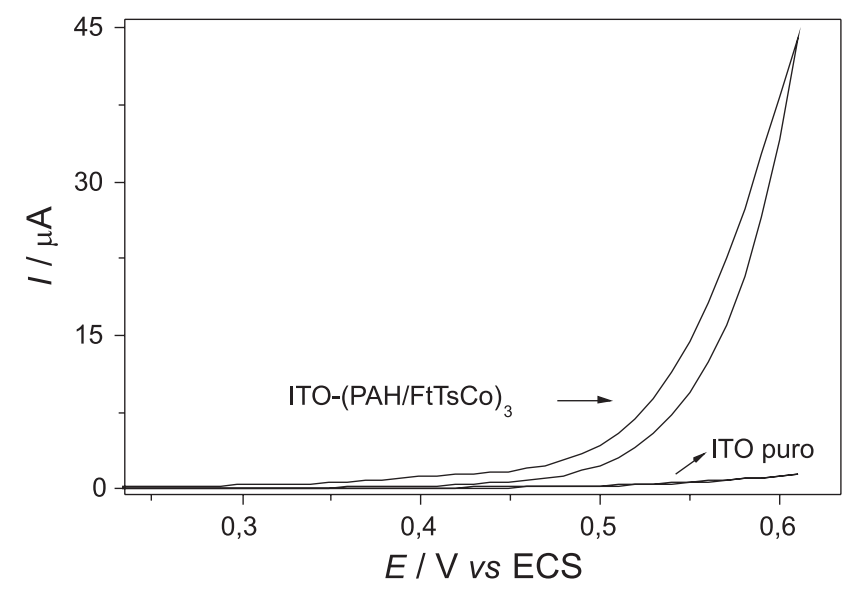

Figura 4S. Performance eletrocatalítica de ITO puro e ITO-(PAH/FtTsCo $)_{3}$ na presença de cisteína em 1,6 $\times 10^{-3} \mathrm{~mol} \mathrm{~L}^{-1}$. Eletrólito PBS - 0,1 mol L $\mathrm{L}^{-1} \mathrm{pH}$ 7,0; velocidade de varredura $=25 \mathrm{mV} \mathrm{s}^{-1}$ e T $=25^{\circ} \mathrm{C}$ 